\title{
CXC chemokine receptor 7 expression in cervical intraepithelial neoplasia
}

\author{
TIAN TANG $^{1}$, QINGJIE XIA ${ }^{2}$ and MINGRONG XI ${ }^{1}$ \\ ${ }^{1}$ Department of Gynecology and Obstetrics, West China Medical School, West China Second University Hospital; \\ ${ }^{2}$ West China Laboratory of Molecular Genetics, Sichuan University, Chengdu, Sichuan 610041, P.R. China
}

Received April 16, 2015; Accepted May 21, 2015

DOI: $10.3892 /$ br.2015.529

\begin{abstract}
Cervical intraepithelial neoplasia (CIN), also known as transformation and dysplasia of cervical intraepithelial cells, is the precancerous lesion of squamous cell carcinoma. CXC chemokine receptor 7 (CXCR7) has been indicated in tumor development and metastasis of multiple malignancies or precancerous lesion. However, the protein expression and function of CXCR7 in different stages of human CIN remains unclear. The present study examined CXCR7 protein expression in cervical tissue samples from 34 patients, including 7 patients with normal cervical tissues (negative control), 10 patients with stage I of CIN (CIN I), 8 patients with CIN II and 9 patients with CIN III. Receiver operating characteristic curves (ROC) were established to evaluate the prognostic value of CXCR7 in differentiating various stages of CIN. Immunohistochemical staining showed that protein expression of CXCR7 was higher in CIN tissues compared with the normal cervical epithelium $(\mathrm{P}<0.05)$. High-grade CIN tissues expressed a higher level of CXCR7 compared to low-grade samples. The ROC curve of integral optical density analysis showed that CXCR7 could discriminate CIN I-III from normal cervical epithelium with $88.9 \%$ sensitivity and $71.4 \%$ specificity, and CIN II-III from the negative control and CIN I with $92.7 \%$ sensitivity and $50.0 \%$ specificity. ROC curve of area analysis also showed that CXCR7 could discriminate CIN I-III from normal cervical epithelium with $70.4 \%$ sensitivity and $100.0 \%$ specificity, and CIN II-III from the negative control and CIN I with $50.0 \%$ sensitivity and $90.0 \%$ specificity. An increase in CXCR7 expression may represent a novel predictor of CIN. The wide expression of CXCR7 in CIN also supports the assumption that CXCR7 plays a role in precancerous lesion progression, as well as proliferation, migration and angiogenesis.
\end{abstract}

Correspondence to: Professor Mingrong $\mathrm{Xi}$, Department of Gynecology and Obstetrics, West China Medical School, West China Second University Hospital, Sichuan University, No. 20 Section 3 Renmin South Road, Chengdu, Sichuan 610041, P.R. China E-mail: qmrjzz@126.com

Key words: cervical intraepithelial neoplasia, CXC chemokine receptor 7, receiver operating characteristics curve analysis, immunohistochemistry

\section{Introduction}

Cervical intraepithelial neoplasia (CIN), also known as transformation and dysplasia of cervical intraepithelial cells, is the precancerous lesion of squamous cell carcinoma. CIN is usually detected through the Papanicolaou (Pap) cytological screening or the high-risk human papillomavirus (HPV) DNA testing (1). However, the Pap test or superior colposcope multipoint sampling requires gynecological surgery. The current inspection method takes time and may fail to collect the most severe lesions. The diagnosis and staging of collected specimens mainly depends on the pathological features.

The mechanisms involved in cell transformation and dysplasia of CIN remain unclear. CXC chemokine receptor type 7 (CXCR7) has been suggested to play a role in tumor development. However, studies on the function of CXCR7 in precancerous lesions are limited. CXCR7 is a non-G protein-coupled receptor. Heterodimerization of CXCR7 with other receptors, such as the epidermal growth factor receptor or CXCR4, triggers intracellular signaling events that are important for the regulation of cell proliferation and apoptosis (2-4). Expression of CXCR7 has been associated with a higher tumor grade and more aggressive tumor growth in different types of cancer, such as cervical cancer, prostate cancer, breast cancer, glioma and hepatocellular carcinoma (5-8). CIN is usually associated with chronic infection of the cervix mainly with the sexually transmitted HPV, particularly the high-risk HPV 16 or HPV 18. The HPV encodes several proteins, including E6 and E7, which lead to the degradation of tumor-suppressor protein p53. In addition, smoking, endogenous and exogenous immunodeficiency are also the risk factors for CIN. CXCR7 was previously reported to play an important role in regulating cell proliferation, apoptosis, tumor evasion, trafficking of lymph node metastasis and metastasis in distinct organs through auto/paracrine mechanisms (9). Additionally, high levels of CXCR7 have been linked to poor disease-free or disease-specific survival rates in cervical cancer patients (5). Progression to invasive cancer occurs in $\sim 1 \%$ of CIN I, $5 \%$ in CIN II and $\geq 12 \%$ in CIN III. Treatment options for higher grade CIN include conization of cervix, hysterectomy, cryocautery, electrocautery and loop electrosurgical excision procedure, which differ from the lower grade CIN. 
The aim of the present study was to determine CXCR7 protein expression in various stages of CIN to understand the potential contribution of CXCR7 in the development and progression of CIN. Receiver operating characteristic (ROC) curves were established to evaluate the prognostic value of CXCR7 in differentiating different stages of CIN, which has not been reported previously.

\section{Materials and methods}

Patient tissues. A total of 34 patients were recruited in the study. Normal cervical tissues obtained from 7 patients with uterine fibroid (with normal cervix) by hysterectomy were used as negative controls. Ten patients with CIN I and 8 diagnosed with CIN II who underwent loop electrosurgical excision procedure were included. A total of 9 patients with CIN III under conization of cervix were also enrolled. The study was approved by the Ethics Committee of West China Hospital of Sichuan University (Sichuan, China). The patient baseline characteristics were anonymously taken to document the process. The Ethics Committees approved this consent procedure.

The clinical stage was determined based on the evaluation of the surgical specimens, which was performed by two independent, experienced pathologists. Assessments were made according to the International Federation of Gynecology and Obstetrics 2009 system.

Immunohistochemistry (IHC). CXCR7 expression in formalin-fixed, paraffin-embedded specimens was assessed by IHC. Tissue blocks were sectioned $(5 \mu \mathrm{m})$ and deparaffinized with xylene. Antigen retrieval with citrate antigen retrieval solution ( $\mathrm{pH}$ 6.0) was performed in a $95^{\circ} \mathrm{C}$ water bath for $40 \mathrm{~min}$. Sections were subsequently rinsed in phosphate-buffered saline and incubated with $3 \% \mathrm{H}_{2} \mathrm{O}_{2}$ for 15 min to block endogenous peroxidase activity.

Each section was incubated with the anti-CXCR7 antibody (1:100; MAB4227) from R\&D Systems (Minneapolis, MN, USA) for $45 \mathrm{~min}$ at $37^{\circ} \mathrm{C}$, followed by labeling with secondary antibody (GK500705; Envision ${ }^{\mathrm{TM}}$ Detection kit; Dako, Glostrup, Denmark). Images of neoplasia and normal tissues were taken at magnification, $\mathrm{x} 400$.

Data analysis. Image-Pro Plus 6.0 software (Media Cybernetics, Rockville, MD, USA) was used for analyzing IHC. For quantitative analysis of the IHC results, area, diameter, density and integral optical density (IOD) were chosen as the measurements. IOD represents the IOD in the whole layer. The area stands for the positive-stained area in the cervical intraepithelial layer. Diameter is also for the positive-stained layer. Density or mean density is equal to IOD SUM/area.

The ROC curves were established to evaluate the prognostic value of CXCR7 in differentiating the stages of CIN. All the statistical analyses were performed using the SPSS 20.0 software (IBM, Corp., Armonk, NY, USA). The statistical significance of CXCR7 correlation in association with the clinicopathological features was assessed using the independent-sample t-test, one-way analysis of variance. $\mathrm{P}<0.05$ was considered to indicate a statistically significant difference.

\section{Results}

CIN samples. The samples were divided into 3 groups according to the degree of atypical hyperplasia: CIN I, atypical cells are confined to the inner $1 / 3$ of the epithelial layer; CIN II, abnormal cells are extended to the $2 / 3$ of the epithelial layer; and CIN III, atypical cells exceed the $2 / 3$ of the epithelial layer, but do not reach the whole layer.

Normal cervical tissues from patients with uterine fibroids were chosen as the negative controls, which showed a limited immunohistochemical reaction (Fig. 1). A relatively low level of CXCR7 expression in CIN I, medium expression in CIN II and high expression in CIN III were observed (Figs. 2-4). Immunohistochemical staining of CIN samples showed that cytoplasm was positively stained in the atypical or dysplastic proliferative cells, particularly in the abnormally arranged cells with abnormal nucleus (Figs. 2-4). In the absence of the primary antibody, no immunoreactivity was detected, indicating the specificity of the antibody used in the study.

Analysis of the CXCR7 expression. Area analysis demonstrated a significantly increased expression of CXCR7 in CIN tissues (151.77 \pm 4.76$)$ compared to normal cervical tissues $(102.52 \pm 32.63)(\mathrm{P}<0.01)$. A significant difference was also observed in IOD analysis between CIN tissues $(23,313.60 \pm 5,052.56)$ and the negative control $(16,266.36 \pm 5,695.51)(\mathrm{P}<0.01)$.

As shown in Table I, the positive area was significantly increased in CIN I, CIN II and CIN III as compared to the negative control $(\mathrm{P}=0.013,0.001$ and 0.008 , respectively). In IOD analysis, a significant difference was observed between the CIN II or CIN III group and the negative control group $(\mathrm{P}=0.02$ and 0.01 , respectively).

To further assess the ability of CXCR7 to distinguish CIN stages, ROC curve analysis was used, which was not reported previously. As shown in Fig. 5, stages I-III of CIN could be differentiated from normal cervical epithelium by CXCR7 staining with an area under the curve (AUC) of 0.825 (IOD) [95\% confidence interval (CI), 0.655 to 0.996 ] and 0.905 (area) (95\% CI, 0.789 to 1.000). For IOD, at the cut-off value of $18,833.945$, the sensitivity and the specificity were 88.9 and $71.4 \%$, respectively, in discriminating CIN I-III from normal tissue (Fig. 5). For area, Fig. 5 showed that, with an AUC of 0.905 (95\% CI, 0.789 to 1.000$)$, CIN I-III could be differentiated from normal cervical epithelium at the cut-off value of 137.515 with sensitivity $(70.4 \%)$ and specificity (100.0\%). Similarly, Fig. 6 showed that CXCR7 could differentiate stages II-III from stage I or negative control with an AUC of 0.663 (IOD) (95\% CI, 0.478 to 0.847 ) or 0.666 (area) (95\% CI, 0.476 to 0.856 ). At the cut-off value of 19,731.52, the sensitivity and specificity were 92.7 and $50.0 \%$ in discriminating CIN II-III from negative to CIN I (Fig. 6). However, CIN III could not be easily distinguished from normal tissue or CIN I-II with an AUC of 0.698 (density) (95\% CI, 0.488 to 0.907, Fig. 7). With the cut-off value of 165.755 , CIN III could be discriminated from normal tissue to CIN I-II at the sensitivity of $50.0 \%$ and the specificity of $90.0 \%$ (AUC of 0.666) (Fig. 7). However, the cut-off value is $19,731.52$ (IOD) with sensitivity (88.9\%) and specificity $(40 \%)$ in discriminating stage III from stages I-II and normal tissue, which was not satisfactory (Fig. 7). 


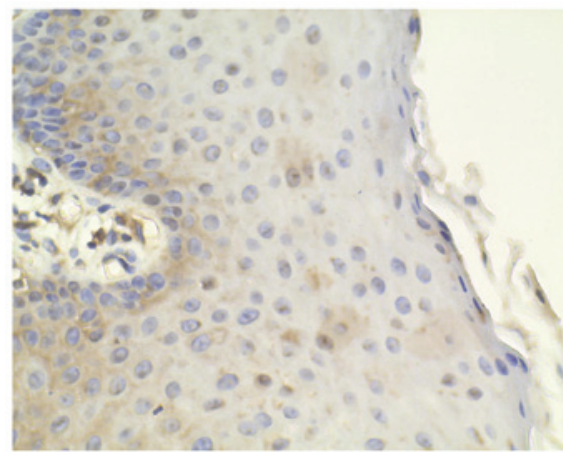

Figure 1. Normal cervical tissues from patients with uterine fibroids were chosen as the negative controls, which show little immunohistochemical reaction (magnification, $\mathrm{x} 400$ ).

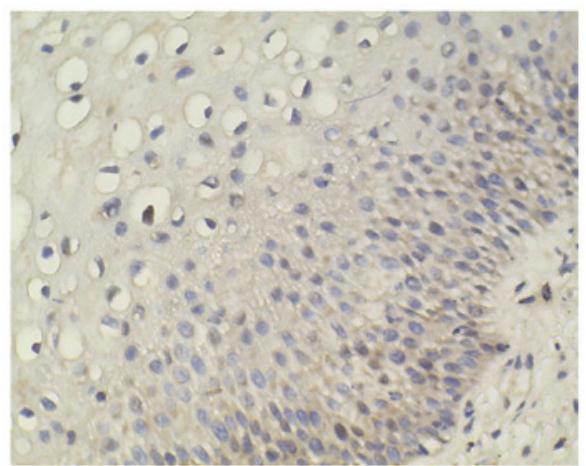

Figure 2. Relatively low level of CXC chemokine receptor 7 protein expression in cervical intraepithelial neoplasia I (magnification, $\mathrm{x} 400$ ).

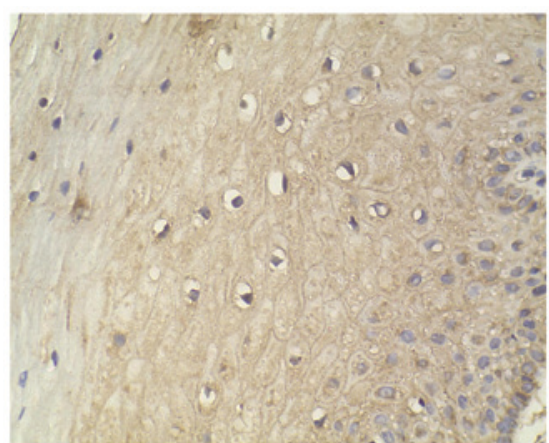

Figure 3. Medium expression level of CXC chemokine receptor 7 staining in cervical intraepithelial neoplasia II (magnification, x400).

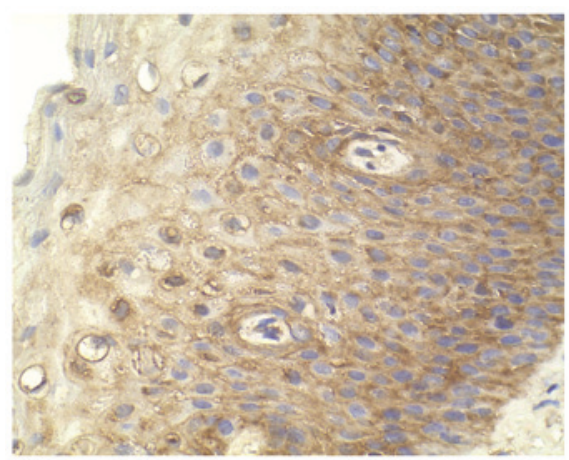

Figure 4. High expression of CXC chemokine receptor 7 staining in cervical intraepithelial neoplasia III (magnification, x400).

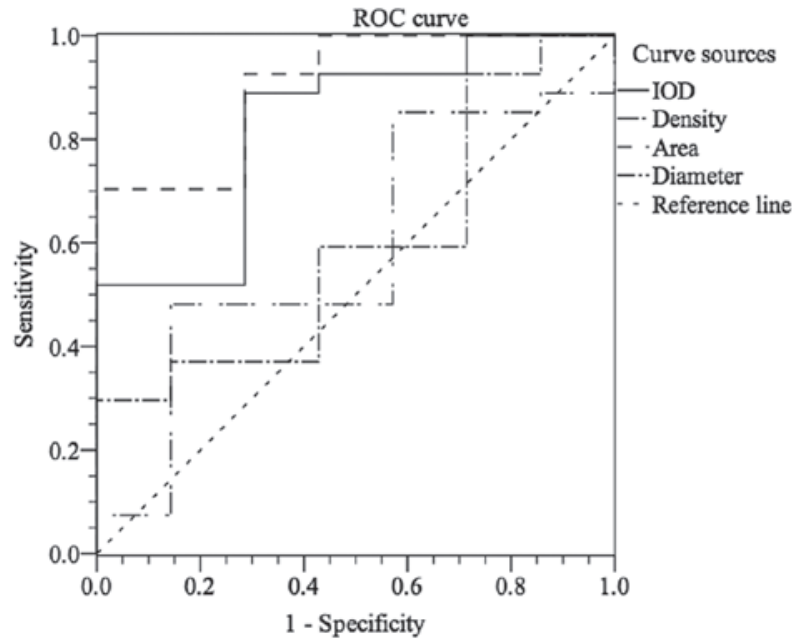

Figure 5. Differentiation of normal tissue from stages I-III of cervical intraepithelial neoplasia by CXC chemokine receptor 7 (CXCR7) expression with receiver operating characteristic (ROC) analysis. IOD, integral optical density.

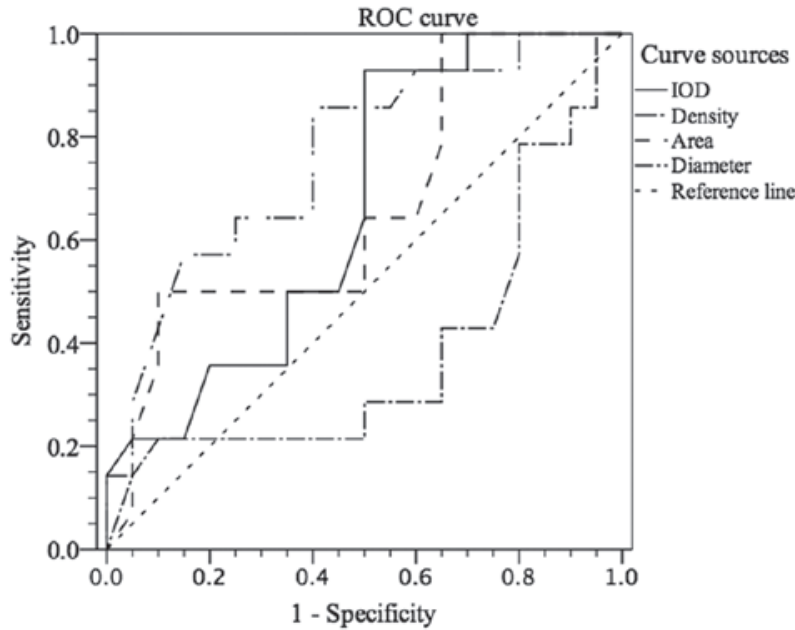

Figure 6. Receiver operating characteristic (ROC) analysis regarding differentiation in normal to cervical intraepithelial neoplasia (CIN) I tissue from CIN II-III tissue by CXC chemokine receptor 7 expression. IOD, integral optical density.

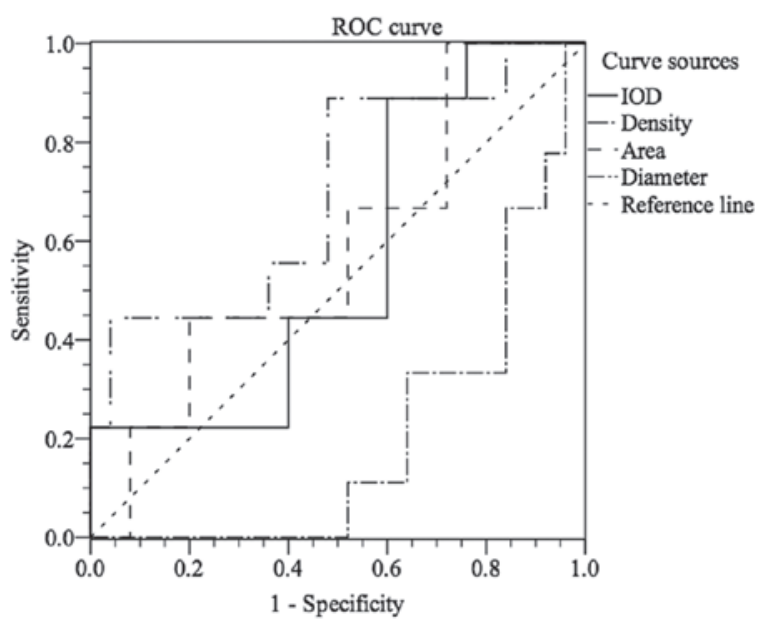

Figure 7. Differentiation of normal to cervical intraepithelial neoplasia (CIN) I-II from CIN III by CXC chemokine receptor 7 expression with receiver operating characteristic (ROC) analysis. IOD, integral optical density. 
Table I. Quantitative analysis of the immunohistochemical results.

Mean difference from

Dependent variables

negative control \pm standard error

P-value

95\% confidence interval

Area

\begin{tabular}{lccc} 
CIN I & $-42.69 \pm 12.96$ & $0.01^{\mathrm{a}}$ & $(-77.92$ to -7.46$)$ \\
CIN II & $-60.80 \pm 13.61$ & $<0.001^{\mathrm{a}}$ & $(-97.80$ to -23.80$)$ \\
CIN III & $-46.27 \pm 13.25$ & $0.01^{\mathrm{a}}$ & $(-82.30$ to -10.24$)$ \\
Density & & & \\
CIN I & $7.71 \pm 5.49$ & 0.51 & $(-7.22$ to 22.63$)$ \\
CIN II & $-5.77 \pm 5.77$ & 0.75 & $(-21.45$ to 9.91$)$ \\
CIN III & $-8.06 \pm 5.61$ & 0.49 & $(-23.33$ to 7.21$)$ \\
Diameter & & & \\
CIN I & $-0.69 \pm 0.50$ & 0.52 & $(-2.06$ to 0.67$)$ \\
CIN II & $-1.40 \pm 0.53$ & 0.06 & $(-2.83$ to 0.04$)$ \\
CIN III & $0.39 \pm 0.51$ & 0.87 & $(-1.01$ to 1.78$)$ \\
IOD & & & $(-11,363.15$ to $-2,153.86)$ \\
CIN I & $-4,604.65 \pm 2,485.56$ & 0.27 & $(-15,409.60$ to $-1,213.90)$ \\
CIN II & $-8,311.75 \pm 2,610.36$ & $0.02^{\mathrm{a}}$ & $(-15,548.61$ to $-1,725.85)$ \\
CIN III & $-8,637.23 \pm 2,541.78$ & $0.01^{\mathrm{a}}$ & \\
\hline
\end{tabular}

${ }^{\mathrm{a}} \mathrm{P}<0.05$. CIN, cervical intraepithelial neoplasia; IOD, integral optical density.

\section{Discussion}

In the present study, the CXCR7 protein was strongly positive in neoplasia. Previously, it was reported that CXCR7 expression was higher in cervical cancer compared to CIN and normal cervical mucosa, particularly in those with an advanced stage of cancer and lymph node metastasis (10). However, the difference in CXCR7 protein expression among different stages of CIN has not been previously investigated and the ROC curve analysis has not been performed. To the best of our knowledge, this is the first study to report that the CXCR7 protein expression level gradually increases from normal cervical intraepithelium to CIN.

It was previously shown with in situ hybridization and IHC that CXCR7 expression is induced in various types of cancer and increased with malignancy $(7,8,11,12)$. The results in the present study that CXCR7 is overexpressed in CIN samples compared with normal tissues support the idea that CXCR7 may serve as a prognostic marker $(5,13)$. Alternatively, the upregulated expression of CXCR7 may favor precancerosis. CXCR7 is expressed in numerous malignant cells, including breast, lung, pancreatic and prostate cancer cells (6,13-15). CXCR7 is expressed in $43 \%$ of cervical cancer specimens and is associated with tumor size and lymph node metastasis together with disease-free survival (5). The significant difference in CXCR7 protein expression level was observed between CIN I, CIN II, CIN III and the negative control in the present study. It is possible that CXCR7 promotes further cancerous activity. CXCR7 is induced under the classic tumor microenvironment (hypoxic and acidic conditions) in human microvascular endothelial cells (8). It is also upregulated in the tumor-associated vasculature $(6,12,16)$. However, the upregulation was not observed in the vasculature of corresponding healthy tissues (12), indicating that angiogenesis is influenced by CXCR7. Hepatocarcinoma cells treated with vascular endothelial growth factor results in a positive feedback for CXCR7 expression (17), further supporting the notion that CXCR7 impacts on vascular growth, therefore favoring precancerosis and subsequently cancerous lesions.

Current evidence suggests that CXCR7 may function in intraepithelial neoplasia dysplasia or cervical interstitial neoplasia. Further investigation is required to understand the accurate mechanism of CXCR7 in regulating precancerosis and to assess the possible value of the CXCR7 expression level detection in the blood samples of patients as a non-invasive, prognostic and diagnostic marker.

\section{Acknowledgements}

The present study was supported by the National Innovative Experimental Projects of Sichuan University, 2012 (grant no. 201210610114). The authors would like to thank Mr.Zengliang Xia and Mr. Faqiang Zhang from the West China Laboratory of Molecular Genetics for their great technical assistance.

\section{References}

1. Origoni M, Salvatore S, Perino A, Cucinella G and Candiani M: Cervical Intraepithelial Neoplasia (CIN) in pregnancy: The state of the art. Eur Rev Med Pharmacol Sci 18: 851-860, 2014.

2. Hartmann TN, Grabovsky V, Pasvolsky R, Shulman Z, Buss EC, Spiegel A, Nagler A, Lapidot T, Thelen M and Alon R: A crosstalk between intracellular CXCR7 and CXCR4 involved in rapid CXCL12-triggered integrin activation but not in chemokine-triggered motility of human $\mathrm{T}$ lymphocytes and CD34+ cells. J Leukoc Biol 84: 1130-1140, 2008. 
3. Décaillot FM, Kazmi MA, Lin Y, Ray-Saha S, Sakmar TP and Sachdev P: CXCR7/CXCR4 heterodimer constitutively recruits beta-arrestin to enhance cell migration. J Biol Chem 286: 32188-32197, 2011

4. Singh RK and Lokeshwar BL: The IL-8-regulated chemokine receptor CXCR7 stimulates EGFR signaling to promote prostate cancer growth. Cancer Res 71: 3268-3277, 2011.

5. Schrevel M, Karim R, ter Haar NT, van der Burg SH, Trimbos JB, Fleuren GJ, Gorter A and Jordanova ES: CXCR7 expression is associated with disease-free and disease-specific survival in cervical cancer patients. Br J Cancer 106: 1520-1525, 2012.

6. Miao Z, Luker KE, Summers BC, Berahovich R, Bhojani MS Rehemtulla A, Kleer CG, Essner JJ, Nasevicius A, Luker GD, et al: CXCR7 (RDC1) promotes breast and lung tumor growth in vivo and is expressed on tumor-associated vasculature. Proc Natl Acad Sci USA 104: 15735-15740, 2007.

7. Hattermann K, Held-Feindt J, Lucius R, Müerköster SS, Penfold ME, Schall TJ and Mentlein R: The chemokine receptor CXCR7 is highly expressed in human glioma cells and mediates antiapoptotic effects. Cancer Res 70: 3299-3308, 2010.

8. Monnier J, Boissan M, L'Helgoualc'h A, Lacombe ML, Turlin B, Zucman-Rossi J, Théret N, Piquet-Pellorce C and Samson M: CXCR7 is up-regulated in human and murine hepatocellular carcinoma and is specifically expressed by endothelial cells. Eur J Cancer 48: 138-148, 2012.

9. Hattermann K and Mentlein R: An infernal trio: The chemokine CXCL12 and its receptors CXCR4 and CXCR7 in tumor biology. Ann Anat 195: 103-110, 2013.

10. Kurban S, Tursun M, Kurban G and Hasim A: Role of CXCR7 and effects on CXCL12 in SiHa cells and upregulation in cervical squamous cell carcinomas in Uighur women. Asian Pac J Cancer Prev 15: 9211-9216, 2014.
11. Shimizu S, Brown M, Sengupta R, Penfold ME and Meucci O CXCR7 protein expression in human adult brain and differentiated neurons. PLoS One 6: e20680, 2011.

12. Madden SL, Cook BP, Nacht M, Weber WD, Callahan MR, Jiang Y, Dufault MR, Zhang X, Zhang W, Walter-Yohrling J, et al: Vascular gene expression in nonneoplastic and malignant brain. Am J Pathol 165: 601-608, 2004.

13. Gebauer F, Tachezy M, Effenberger K, von Loga K, Zander H, Marx A, Kaifi JT, Sauter G, Izbicki JR and Bockhorn M: Prognostic impact of CXCR4 and CXCR7 expression in pancreatic adenocarcinoma. J Surg Oncol 104: 140-145, 2011.

14. Burns JM, Summers BC, Wang Y, Melikian A, Berahovich R, Miao Z, Penfold ME, Sunshine MJ, Littman DR, Kuo CJ, et al: A novel chemokine receptor for SDF-1 and I-TAC involved in cell survival, cell adhesion, and tumor development. J Exp Med 203: 2201-2213, 2006

15. Wang J, Shiozawa Y, Wang J, Wang Y, Jung Y, Pienta KJ, Mehra R, Loberg R and Taichman RS: The role of CXCR7/RDC1 as a chemokine receptor for CXCL12/SDF-1 in prostate cancer. J Biol Chem 283: 4283-4294, 2008.

16. Sánchez-Martín L, Estecha A, Samaniego R, Sánchez-Ramón S, Vega MÁ and Sánchez-Mateos P: The chemokine CXCL12 regulates monocyte-macrophage differentiation and RUNX3 expression. Blood 117: 88-97, 2011

17. Zheng K, Li HY, Su XL, Wang XY, Tian T, Li F and Ren GS: Chemokine receptor CXCR7 regulates the invasion, angiogenesis and tumor growth of human hepatocellular carcinoma cells. J Exp Clin Cancer Res 29: 31, 2010 